\title{
Nonlinear Dynamics in Optoelectronics Structures with Quantum Well
}

\author{
Andreea Rodica Sterian \\ Additional information is available at the end of the chapter \\ http://dx.doi.org/10.5772/intechopen.74557
}

\begin{abstract}
The author presents some results on nonlinear dynamics in optoelectronics nanostructures as lasers with quantum wells and quantum well solar cells using mathematical modeling and numerical simulations of the phenomena which take place in such kinds of structures. The nonlinear dynamics takes the complexity of the phenomena into account, which govern the field-substance interaction. Computational software was elaborated to study the nonlinear phenomena in such quantum devices, which put into evidence their complex nonlinear dynamics, characterized by bifurcation points and chaos, and the critical values of the parameters being determined. The mathematical modeling and numerical simulations for the quantum well solar cells for optimizing the values of their optical parameters (refraction index, reflectance, and absorption) were also analyzed, so that the conversion efficiency of the devices can be improved. Although in our study we have considered only rectangular quantum wells, the hybrid model allows computing the optimum values of the parameters whatsoever the form of the quantum wells. The developed numerical models and the obtained results are consistent with the existing data in the literature for the optoelectronics of quantum well structures, having important implications in the applications.
\end{abstract}

Keywords: quantum well laser, quantum well solar cell, hybrid model, bifurcation diagram, chaotic state, quantum engineering, chaos masking

\section{Introduction}

The nonlinear dynamics is treated taking into account the complexity of the phenomena which govern the field-substance interaction including the dissipative phenomena [1-6]. Computational software was elaborated to study the nonlinear phenomena in such kinds of quantum devices [7-9]. 
A quantum well (QW) structure improves the functioning characteristics of laser diodes and of the solar cells as the emitted wavelength depends on nanostructure dimension (quantum size effect) $[3,10-13]$. Such a structure is one in which the active region of the device is so narrow that quantum confinement occurs, according to quantum mechanics. The wavelength of the light emitted by a quantum well laser is determined by the width of the active region rather than just the band gap of the material from which the device is realized. Consequently, much shorter wavelengths can be obtained from quantum well lasers than from conventional laser diodes using a particular semiconductor material. The realization of quantum well structures represents cutting-edge technological advances in the field of semiconductor optoelectronics structures. These devices, unlike the classic ones, have the active area in the form of a periodic structure of semiconductor layers having different forbidden energy bands. These layers have very small thicknesses (nanometer units), their deposition being possible using advanced technologies and special deposition techniques.

In this chapter, first, we shall recall different forms of the rate equations used to take into account the complexity of the phenomena which govern the field-substance interaction. Based on computational software which was elaborated for the rate equation models presented, the phenomena were numerically studied in such a kind of quantum devices, and the utility of these models on some very simple examples and applications by different methods of simulations [14-21] was illustrated.

Second, we treat the complex nonlinear dynamics, characterized by bifurcation points and chaos, the critical values of the parameters being determined. The work emphasizes the variety of dynamical modes of operation of a semiconductor laser as a result of modulation of the injection current [22-28].

The third part of the chapter is dedicated to the particularities of the quantum well solar cells. The simulation results for the optical parameters (refraction index, reflectance, absorption coefficient) are presented as well as those for the optimization of the quantum efficiency of QW solar cells [21, 29-34].

Finally, as the application of the chaotic dynamics, an optical communications channel with chaotic laser signals is presented to demonstrate the important implications of these kinds of systems in quantum engineering and for transmission and processing of the optical information [35-40].

\section{Modeling methods and techniques for quantum well lasers}

\subsection{Standard rate equations}

Several levels of descriptions and modeling of field-substance interaction are known in the theory of lasers, depending on the classical or quantum character of the evolution equations, describing the two subsystems in interaction, the substance and the electromagnetic field, the laser system being a dissipative structure which takes on self-organizing phenomena, far from 
equilibrium. Besides the phenomenological description of lasers owing to Einstein, who discovered the stimulated emission in 1016, which makes the amplification of coherent radiation in lasers possible, the theories developed for laser phenomena description are the thermodynamic theory, based on the equations of the rates as equations of balance for the populations of the laser levels and the density of the photons in the laser cavity, considering the dissipation; the semi-classical theory (or semi-quantum) in which the population equations are quantum, established as motion equations for the quantum operators corresponding to the populations of the levels involved and the field equation which is the classical equation describing the electromagnetic field in the laser medium, polarization of the medium being the source of the field; and the quantum theory in which both the substance and the electromagnetic field are described by quantum equations of evolution. The abovementioned theories are complementary to each other and are used according to the phenomena whose description is being followed in the studied issue or application.

As a system, the laser is described by the equations of two nonlinear coupled oscillators, a quantum oscillator represented by the polarization of the active medium and a classical oscillator which is the electromagnetic field from the optical resonant cavity. By pumping, the system receives external energy to realize the population inversion. Above the threshold, the oscillation condition being accomplished, the energy accumulated in the active medium is liberated through stimulated emission, under the form of the coherent light beam of laser.

The main quantum well laser model used in this chapter is based on the description of semiconductor lasers using the thermodynamic theory of the rate equations [14].

While the first models were based on one pair of equations to describe the density of photons and carriers in the active region, recent approaches include additional rate equations to take into account, and carriers transport between the active region and the adjacent layers of the structure as in Ref.s [15-18].

It can be observed that in most cases, the rate equations lead to multiple solutions, although only one solution is correct. Javro and Kang [19] showed that incorrect solutions or without physical sense can be eliminated or avoided through a change of variables in the rate equations. However, the transformations used are available under certain conditions, and for some cases, they give unrealistic solutions. These shortcomings are caused mainly due to the linear character of the gain-saturation coefficient. A more general expression of the gain-saturation coefficient, proposed by Channin, can be used to obtain models for operation regimes having a solution unique. Agrawal suggests another expression for this coefficient, which is also suitable. As is shown, any of these two forms of the gain-saturation coefficient can be used to obtain models with a solution unique to the operation regime.

\subsubsection{The model with linear gain saturation}

One of the prevailing laser diode models is based on a set of rate equations. The rigorous derivation of these equations originates from Maxwell equations with a quantum mechanical approach for the induced polarization. However, the rate equations could also be derived by considering physical phenomena described as in [3]. 
The population equation is as follows:

$$
\frac{d N}{d t}=\frac{I}{q V_{a c t}}-g_{0}\left(N-N_{0}\right)(1-\varepsilon S) S-\frac{N}{\tau_{p}}+\frac{N_{e}}{\tau_{n}}
$$

Similarly, the photon density equation is written as.

$$
\frac{d S}{d t}=\Gamma g_{0}\left(N-N_{0}\right)(1-\varepsilon S) S+\frac{\Gamma \beta N}{\tau_{n}}-\frac{S}{\tau_{p}}
$$

The photon density $S$ reported the output power $P f$ as described by Eq. (3):

$$
\frac{S}{P_{f}}=\frac{\Gamma \tau_{p} \lambda_{0}}{V_{a c t} \eta h c}=\vartheta
$$

Eq. (1) relates the rate of change in carrier concentration $N$ to the drive current $I$, the carrier combination rate and the stimulated-emission rate $S$. Eq. (2) relates the rate of change in photon density $S$ to photon loss, the rate of coupled recombination into the lasing mode, and the stimulated-emission rate. The photon density $S$ to the output power $P_{f}$ is described by Eq. (3). The other parameters used have well-known significances as in Ref. [14]. This simple model can be directly implemented with Matlab Simulink.

\subsubsection{The model with nonlinear gain saturation}

An alternate version of the standard rate equations ensures us that for a nonnegative injection current, one nonnegative solution exists for density of both carriers and photons. The equations treated henceforth are the more generalized versions of Eqs. (1) and (2) with the linear gain-saturation term replaced by the term proposed by either Channin or Agrawal. The new equations are shown below as in Ref. [20]:

$$
\begin{gathered}
\frac{d N}{d t}=\frac{\eta_{i} I}{q N_{w} V_{a c t}}-R_{w}(N)-\Gamma_{c} v_{g r} \frac{\alpha(N)}{\Phi(S)} S \\
\frac{d S}{d t}=-\frac{S}{\tau_{p}}+N_{w} R_{w \beta}(N)+N_{w} \Gamma_{c} v_{g r} \frac{\alpha(N)}{\Phi(S)} S \\
\frac{S}{P_{f}}=\frac{\lambda \tau_{p}}{\eta_{c} V_{a c t} h c}=\vartheta
\end{gathered}
$$

Eq. (4) relates the rate of change in carrier concentration $N$ with the injection current, the carrier recombination rate and the stimulated-emission rate. In order to account for different recombination mechanisms, it considers $R_{w}(N)=A N+B N^{2}+C N^{3}$, where $A, B$, and $C$ are the unimolecular, radiative, and Auger recombination coefficient, respectively. Eq. (5) relates the rate of change in photon density to photon loss, the rate of coupled recombination into the lasing mode, and the stimulated-emission rate. In the above equations, the two gain-saturation terms are 


$$
\begin{aligned}
& \alpha(N)=G_{0} \ln \left(\frac{R_{w}(N)}{R_{w}\left(N_{0}\right)}\right) \\
& \alpha(N)=g_{l} G_{0}\left(\frac{N}{N_{0}}-1\right)
\end{aligned}
$$

where $G_{0}$ is the gain coefficient per quantum well, $N_{0}$ is the optical transparency density, and $g_{l}$ is a factor obtained when linearizing the logarithmic gain around $N_{0}$. Specifically,

$$
g_{l}=\frac{A N_{0}+2 B N_{0}^{2}+3 C N_{0}^{3}}{A N_{0}+B N_{0}^{2}+C N_{0}{ }^{3}}
$$

The gain-saturation function can take on one of the following two forms:

$$
\begin{gathered}
\phi^{-1}(S)=\frac{1}{1+\varepsilon \Gamma_{c} S} \\
\phi^{-1}(S)=\frac{1}{\sqrt{1+\varepsilon \Gamma_{c} S}}
\end{gathered}
$$

Using the approach taken in [11, 18, 20], an equivalent circuit model based on the above equations can be implemented in SPICE. Unlike models based on the rate equations that use a linear gainsaturation term, this circuit model is applicable for all nonnegative values of injection current.

\subsection{Numerical experiments in MathCad}

A comparative study of the models presented above for quantum well lasers is possible by numerical integration in MathCad of the corresponding equations to find the response for different types of pumping signals. The model with linear gain saturation, given by Eqs. (1) and (2), was integrated for a constant injection current, the corresponding waveforms for the optical output power being illustrated in [10]. The nonlinear gain-saturation model of Eqs. (4) and (6) was integrated for an injection current rectangular (Figure 1), which for some periods can be written under the form.

$$
\begin{aligned}
I_{i}(t)= & 10^{-8}+0,01+0,25 I_{i}\left(t-2 \cdot 10^{-9}\right)+ \\
& +0,25 I_{i}\left(t-7 \cdot 10^{-9}\right)+0,25 I_{i}\left(t-12 \cdot 10^{-9}\right) .
\end{aligned}
$$

In general, all models studied give satisfactory results for the study of the transient and dynamic regime at low injection levels. At high injection rates, the numerical results obtained show the specific limits of each model, being consistent with the theoretical analysis.

\subsection{Methods of modeling solutions of the rate equations}

In this section, the two methods of modeling the solutions of the rate equations analyzed in [14] are presented. The first modeling technique is based on standard rate equations with a set 


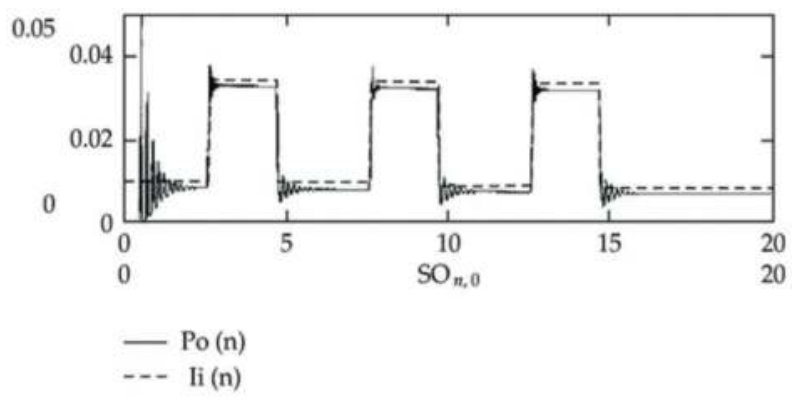

Figure 1. The waveforms of optical output power for an injection current rectangular in the model with nonlinear gain saturation.

of parameters given directly in Simulink. The second model is based on the standard rate equations that use a gain-saturation term and can be implemented in SPICE.

\subsubsection{The Simulink modeling technique}

This simple model can be directly implemented with Simulink like in Figure 2.

The input parameter from a signal generator is $I$, while $S, N$ and $P f$ are the output parameters. All the parameters in the rate equations can be modified before the simulation starts as in all specialized papers [14-16].

With the above model, different signals can be used as input current for the quantum well laser. They show that the theoretical response of the equations is good when compared with real results that are expected in applications. Signals like saw tooth and sine types are used as input. The results are shown in Figure 3. They show very fast response at a low level of the current. Low threshold current is the main feature of quantum well lasers, and it is directly shown for the basic form of rate equation. The simulation is not perfect, and this is because of negative solutions for $N$ and $S$ and high power solution of the equations.

\subsubsection{The SPICE modeling technique}

In an alternate version of the rate equations, the linear gain-saturation term is replaced by the term proposed by either Channin or Agrawal [10, 15, 20]. Using the approach taken in [11, 14], an equivalent circuit model based on the above equations can be implemented in SPICE. Unlike models based on the rate equations that use a linear gain-saturation term, this circuit model is applicable for all nonnegative values of injection current.

In Figure 4, the circuit implementation is shown. This equivalent circuit can be obtained through suitable handling of the rate equations and by the transformations of variables. Diodes D1 and D2 and current sources Ic1 and Ic2 are modeling the linear recombination and charge storage in the device, while Br1 and Bs1 are modeling the effects of additional recombination mechanisms and stimulated emission, respectively, on the charges carrier density. 


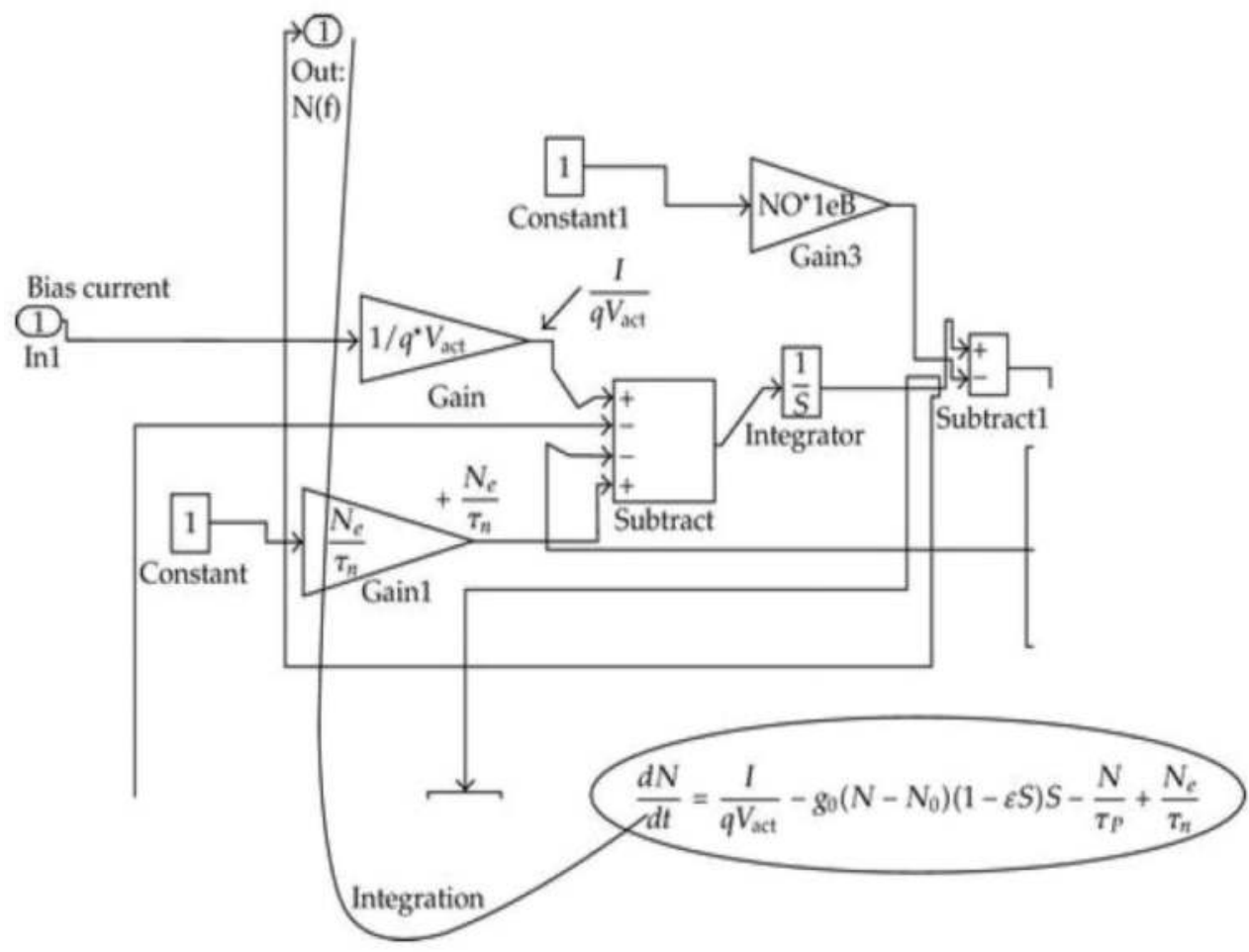

Figure 2. The Simulink block scheme of the rate equations.

The components Rph and Cph of the circuit help to model the time variation of the photon density under the effects of spontaneous and stimulated emission, which are accounted for by $\mathrm{Br} 2$ and Bs2, respectively. Finally, the source Bpf produces the optical output power of the laser in the form of a voltage. The circuit equations are given as follows:

$$
\begin{gathered}
I=I_{T 1}+I_{D 1}+I_{C 1}+B_{r 1}+B_{s 1} \text { where } I_{T 1}=I_{D 1}+I_{C 1} \\
2 \tau_{p} \frac{d m}{d t}+m=B_{r 2}+B_{s 2} \text { and } B_{p f}=(m+\delta)^{2} \\
I_{D 1}=\frac{q N_{w} V_{a c t} N_{e}}{2 \eta_{i} \tau_{n}}\left[\exp \left(\frac{q V}{n k T}\right)-1\right] \\
I_{D 2}=\frac{q N_{w} V_{a c t} N_{e}}{2 \eta_{i} \tau_{n}}\left[\exp \left(\frac{q V}{n k T}\right)-1+\frac{2 q \tau_{p}}{n k T} \exp \left(\frac{q V}{n k T}\right) \frac{d V}{d t}\right] \\
I_{c 1}=I_{c 2}=\frac{q N_{w} V_{a c t} N_{e}}{2 \eta_{i} \tau_{n}}
\end{gathered}
$$



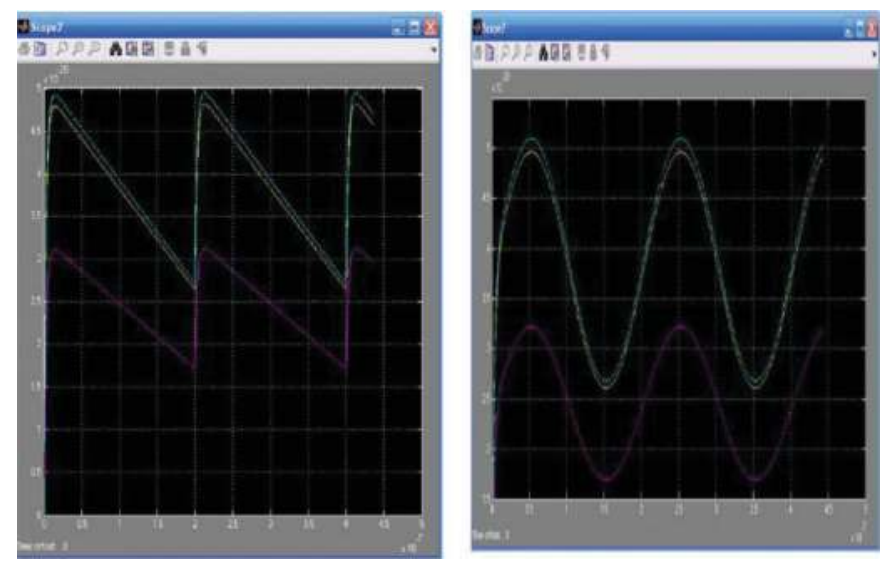

Figure 3. Output for saw tooth input signal of $10 \mathrm{~mA}$ amplitude and frequency of $5 \mathrm{MHz}$, and the output for the input signal sinusoidally having the same values for the amplitude and frequency.

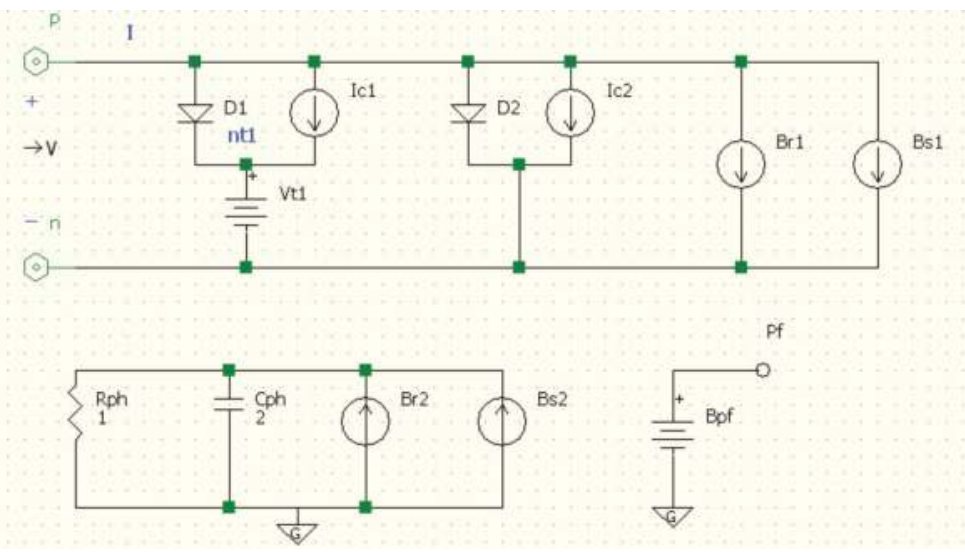

Figure 4. The electric circuit corresponding to the rate equation model.

$$
\begin{gathered}
B_{r 1}=\frac{q N_{w} V_{a c t}}{\eta_{i}} R_{\omega 2}\left(\Theta I_{T 1}\right) \\
B_{s 1}=\frac{\lambda \tau_{p} N_{w} \Gamma_{c} v_{g r}}{\eta i \eta_{c} h c} \frac{\alpha\left(\Theta I_{T 1}\right)}{\phi\left(\vartheta(m+\delta)^{2}\right)} \vartheta(m+\delta)^{2} \\
B_{r 2}=\frac{N_{w} \eta_{c} V_{a c t} h c}{\lambda \vartheta(m+\delta)} R_{\omega 2}\left(\Theta I_{T 1}\right) \\
B_{s 2}=\tau_{p} N_{w} \Gamma_{c} v_{g r} \frac{\alpha\left(\Theta I_{T 1}\right)}{\phi\left(\vartheta(m+\delta)^{2}\right)}(m+\delta)-\delta
\end{gathered}
$$




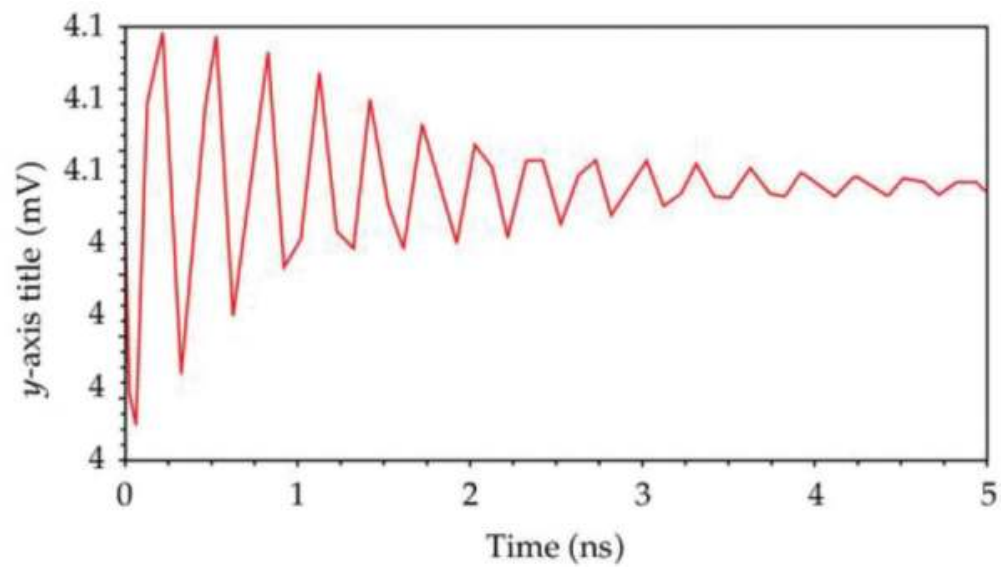

Figure 5. Transient output power response between 0 and $5 \mathrm{~ns}$.

We implemented the SPICE netlist in AIM-SPICE, but calculating the parameters in the netlist is time-consuming. Figure 5 shows results from PSPICE simulation with simple DC sweep and transient output power in response. (Obs: output power is given in Volt, because SPICE cannot have output variables in Watt).

Modifying one parameter will result in new calculations and new SPICE netlist. Simulations are not limited to SPICE, any all-purpose circuit simulators can be used to get similar results. Future development software that integrates circuit simulation and other modeling methods for quantum well lasers can be built to have a tool that models these devices from all points of view.

As a future development, we mention the method of the MATH package Simulink to simulate the behavior of the quantum well laser diodes with distributed feedback using the rate equations [16].

A more deeper additional can be the finite-difference time-domain (FDTD) method and then all are bundled in one software package for more simulation options [34].

\section{Nonlinear dynamics of the quantum well laser}

\subsection{Modulation of the quantum well laser}

We study the amplitude modulation of the injection current [22, 23]:

$$
I=I_{b}[1+m \sin (2 \pi f t)]
$$

where $I_{b}$ is the polarization current and $m$ modulation index: 


$$
m=\frac{I_{m}}{I_{b}}
$$

where $I_{m}$ is the amplitude of the modulating signal $\left(i_{m}=I_{m} \sin (\omega t)\right), f$ being the modulation frequency.

\subsection{The model of small signal and the frequency response}

In this section, we analyze the low signal model and the frequency response of the laser for different polarization currents. The small signal model can be obtained from the equations of the rates, with two equations of populations [10], replacing $I, N_{b}, N_{w}$, and $S$ with $N_{b 0}+\Delta N_{b} e^{j \omega t}, I_{b}+\Delta I e^{j \omega t}, N_{w 0}+\Delta N_{w} e^{j \omega t}$, respectively, $S_{0}+\Delta S e^{j \omega t}$, where $I_{b}$ is the polarization current. The quantities $N_{b 0}, N_{w 0}, S_{0}$ are the solution of the considered rate equations, when the laser is pumped with the polarization current $I_{b}, \Delta I$ is the amplitude of a small perturbation overlaid on $I_{b}$ and $\Delta N_{b}, \Delta N_{w}, \Delta S$ are the amplitudes of population densities and photon density corresponding to a small excitation. The frequency response of the laser is represented graphically in Figure 6 using system (24) where $N_{w 0}$ and $S_{0}$ are calculated by means of the large signal model, placing $I(t)=I_{b}$ for the large $t$. The simulation parameters can be found in [10]

$$
\begin{aligned}
& \left(\begin{array}{l}
-\frac{i \Delta I}{q V_{w}} \\
0 \\
0
\end{array}\right)= \\
& =\left(\begin{array}{lll}
\bar{\omega}-\frac{i}{\tau_{c a p}}-\frac{i}{\tau_{n}} & \frac{i}{\tau_{e s c}} \\
\frac{i}{\tau_{c a p}} & \omega-\frac{i}{\tau_{e s c}}-\frac{i}{\tau_{n}}-i g_{0} \frac{S_{0}}{1+\varepsilon S_{0}} & -i \frac{g_{0}}{\left(1+\varepsilon S_{0}\right)^{2}}\left(N_{w 0}-N_{0}\right) \\
0 & i \Gamma g_{0} \frac{S_{0}}{1+\varepsilon S_{0}}+i \Gamma \frac{\beta_{s p}}{\tau_{n}} & \bar{\omega}+i \Gamma \frac{g_{0}}{\left(1+\varepsilon S_{0}\right)^{2}}\left(N_{w 0}-N_{0}\right)-\frac{i}{\tau_{p}}
\end{array}\right) \cdot\left(\begin{array}{l}
N_{b} \\
N_{w} \\
S
\end{array}\right)
\end{aligned}
$$

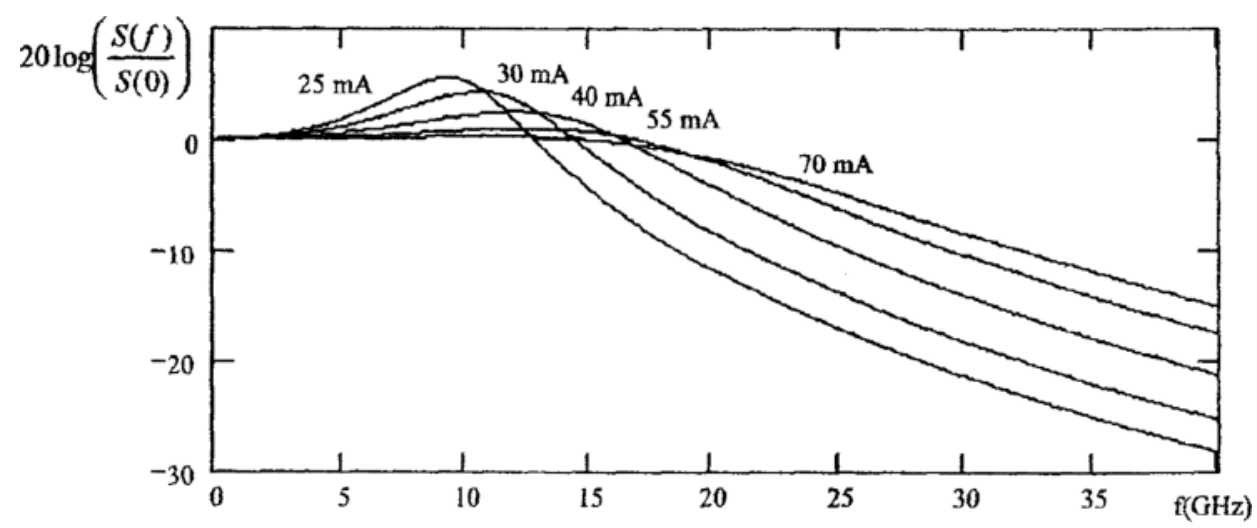

Figure 6. Frequency response of QW laser for $I_{b}=25 ; 30 ; 40 ; 55 ; 70 \mathrm{~mA}$. 
In the preceding paragraph, a small signal pattern was derived to analyze the frequency response of a QW laser for different polarization currents $I_{b}$. By increasing this current in a certain range, the laser band, the maximum modulation frequency increases.

As shown in the subsequent text, the QW laser band can be further extended if the gain of the active medium $g_{0}$ (e.g., carrier temperature in the active region of the laser) is modulated additionally to $I$. For this purpose, a new low signal model is derived. This time, we use the equations of the large signal pattern with one population equation [10, 20]. Optical gain is no longer a constant, having the form $g(t)=g_{0}(1+\Delta P \sin \omega t)$, where $\Delta P$ represents a small fraction of $g_{0}$. The system of linear equations of the small signal pattern written in a matrix form easily implementable in MathCad has the form:

$$
\begin{aligned}
& \left(\begin{array}{l}
\frac{-i \Delta I}{q V_{a c t}}+i(1-\varepsilon S) S g_{0}\left(N-N_{0}\right) \Delta P \\
-i(1-\varepsilon S) S g_{0} \Gamma\left(N-N_{0}\right) \Delta P
\end{array}\right)= \\
& =\left(\begin{array}{ll}
\bar{\omega}-i g_{0} S(1-\varepsilon S)-\frac{i}{\tau_{n}} & -i(1-2 \varepsilon S) g_{0}\left(N-N_{0}\right) \\
i \Gamma g_{0} S(1-\varepsilon S)+i \Gamma \beta \frac{1}{\tau_{n}} & \bar{\omega}+i(1-2 \varepsilon S) g_{0} \Gamma\left(N-N_{0}\right)-\frac{i}{\tau_{p}}
\end{array}\right) \cdot\left(\begin{array}{c}
N \\
S
\end{array}\right) .
\end{aligned}
$$

The results obtained by simulations for the photon density correspond to the three modulation cases and are given in [10]: (1) modulation of injection current and simultaneously of g; (2) the simple modulation of $I$; and (3) simple modulation of $\mathrm{g}$, being presented in Figure 7 (in all cases, $I_{b}=50 \mathrm{~mA}$ ).

An increase in the bandwidth of the laser is observed when both $I$ and $g_{0}$ are simultaneously modulated.

\subsection{Routes to chaos and bifurcation diagrams}

In Figure 8, the variation of the photon density $S$ for different modulation indices as in [28] is compared.

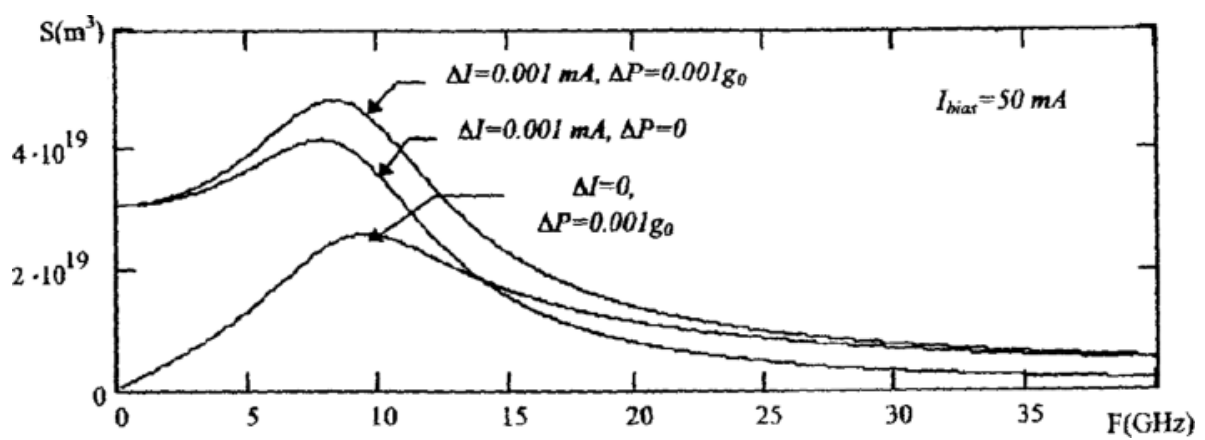

Figure 7. The photon density for three modulation cases: (1) modulation of injection current and simultaneously of g; (2) the simple modulation of $I$; and (3) simple modulation of $\mathrm{g}$. 


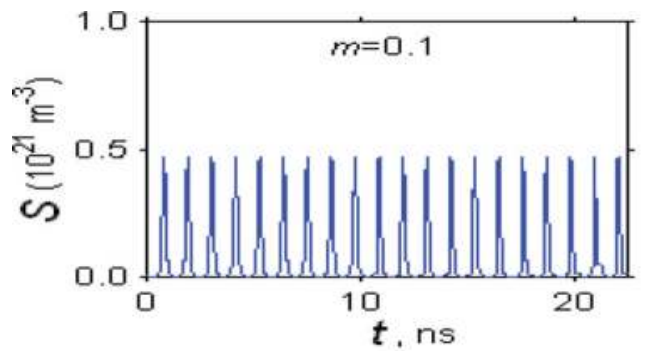

a)

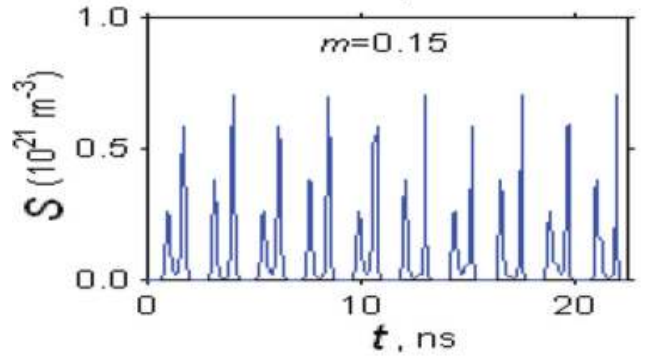

c)

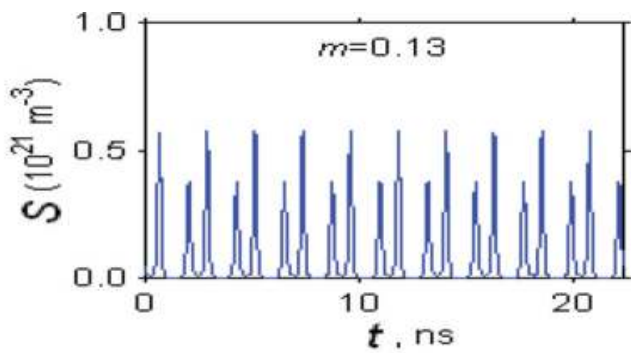

b)

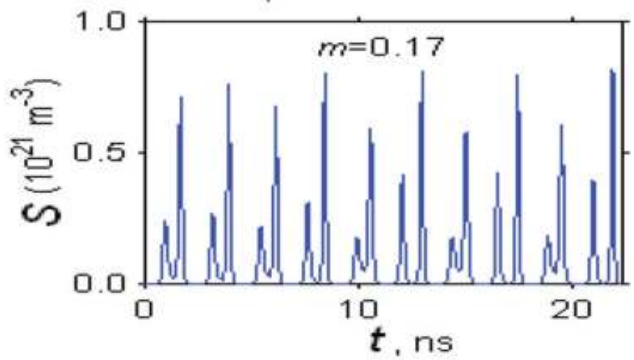

d)

Figure 8. Time dependence of photon density for different modulation indices. The $1 \mathrm{~T}$ periodic signal in (a) becomes a double period (2 T) in (b), with the $4 \mathrm{~T}$ period in (c) and chaotically in (d).

Such a route to chaos with the increase of $m$ by doubling of the period can also be presented qualitatively in the phase space as in $[10,28]$. Knowing the hierarchy of instabilities of laser devices is useful in designing and calculating stable operating regimes in applications. Next, we analyze the bifurcation diagram of the peak photon density according to the modulation index (Figure 9) in the direct sense (with the increase of $m$ ) and vice versa (with the decrease of $m$ ) for a modulation cycle as an indicator of the evolution of the system.

In the chaotic region, there are periodic dynamic windows and a hysteresis dynamics (marked with arrows in Figure 9). In Figure 10, another bifurcation diagram is presented for a modulation frequency $f=f_{0} / 2$, the other parameters remaining unchanged.

This result shows the complexity of system dynamics, without this complexity to be fully elucidated to date [12].

\subsection{Rate equations with noise sources}

The noise sources $F_{n}$ and $F_{s}$ are considered in the rate equations as in [27, 28]:

$$
\begin{gathered}
\frac{\mathrm{d} N}{\mathrm{~d} t}=\frac{I}{q V_{a c t}}-g_{0}\left(N-N_{0}\right) S-\frac{N}{\tau_{n}}+F_{n}(t) \\
\frac{\mathrm{d} S}{\mathrm{~d} t}=g_{0}\left(N-N_{0}\right) S-\frac{S}{\tau_{p}}+\gamma \frac{N}{\tau_{n}}+F_{S}(t)
\end{gathered}
$$




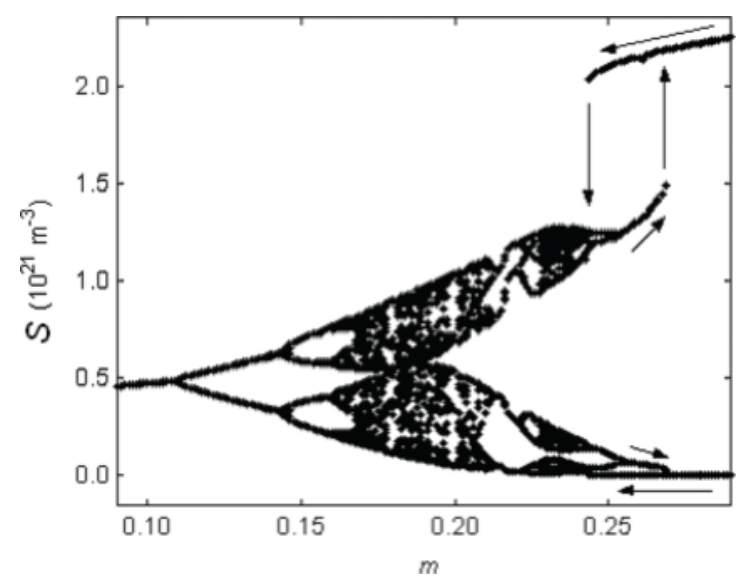

Figure 9. Bifurcation diagram of photon density depending on the modulation index.

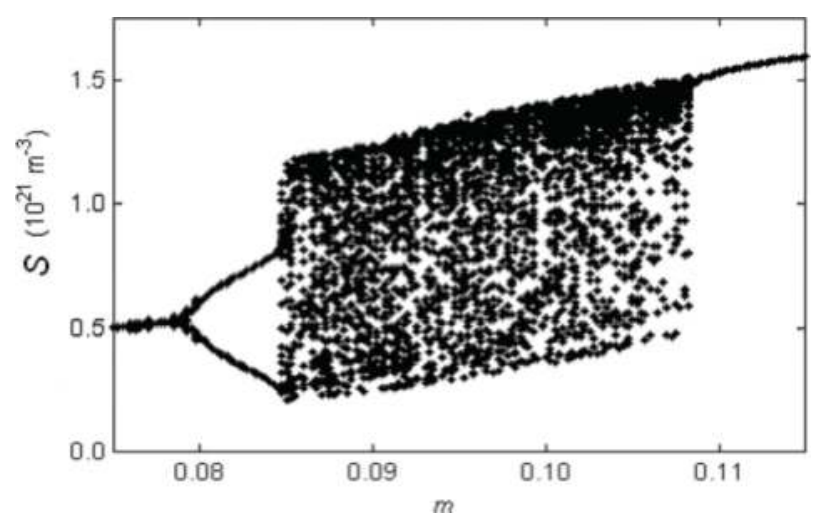

Figure 10. Bifurcation diagram for $f=f_{0} / 2$.

In the Markoff approximation, noise sources are Gaussian variables of zero average, $\delta$ correlated, as

$$
\begin{gathered}
\left\langle F_{\mathrm{n}}(t)\right\rangle=0, \quad\left\langle F_{\mathrm{n}}(t) F_{\mathrm{n}}\left(t^{\prime}\right)\right\rangle=V_{\mathrm{n}}^{2} \delta\left(t-t^{\prime}\right) \\
\left\langle F_{\mathrm{s}}(t)\right\rangle=0, \quad\left\langle F_{\mathrm{s}}(t) F_{\mathrm{s}}\left(t^{\prime}\right)\right\rangle=V_{\mathrm{s}}^{2} \delta\left(t-t^{\prime}\right) \\
\left\langle F_{\mathrm{n}}(t) F_{\mathrm{s}}\left(t^{\prime}\right)\right\rangle=r V_{\mathrm{n}} V_{\mathrm{s}} \delta\left(t-t^{\prime}\right)
\end{gathered}
$$

In the above expressions, $V_{n}^{2}$ and $V_{s}^{2}$, there are variances of the random variables $F_{n}$ and $F_{s}$, respectively, and $r$ a correlation coefficient. See reference [28] for details. In the numerical modeling of Eqs. (26) and (27), it considers, for the intervals $\Delta t$, the autocorrelation function (7.81) of the form: 


$$
\left\langle F_{\mathrm{n}}(t) F_{\mathrm{n}}\left(t^{\prime}\right)\right\rangle=\left\{\begin{array}{lll}
V_{\mathrm{n}}^{2} / \Delta t, & \text { pentru } & \left|t-t^{\prime}\right|<\Delta t \\
0, & \text { pentru } & \left|t-t^{\prime}\right|>\Delta t
\end{array}\right.
$$

This condition leads to the representation of

$$
F_{n}=\left(V_{n} / \sqrt{\Delta t}\right) x_{n}
$$

where $x_{n}$ is a Gaussian random variable of zero average and unit variance.

By using Gaussian random numbers supplied by MATLAB functions, integration is achieved in much smaller steps $\Delta t$ relative to the modulation period. Numerical calculations show that no matter how small the noise is, it disturbs the attractors and can even produce transitions between coexisting attractors.

For a modulation index $m=0,265$, two stable attractors coexist (Figure 11a and b), but the presence of noise induces transitions from one attractor to another as is shown in Figure 11c. See references $[27,28]$.

The complexity of phenomena taking place in a laser diode in the presence of modulation is correlated with the nonlinearity of the stimulated-emission rate $g_{0}\left(N-N_{0}\right) S$, which will be analyzed extensively in the following paragraph.

\subsection{Nonlinear dynamics of the MQW: A case study}

This section presents a case study in which the nonlinear dynamics of the quantum hole laser is systematically analyzed on the basis of two population equations proposed by Nagarajan et al. [26]. In this model, a quantum well structure is modeled as a single quantum well structure, "concentrating" both the quantum wells and the barriers together.

Rate equations were solved numerically. The simulations were performed in the Matlab programming language using a fourth-order Runge-Kutta scheme [10].
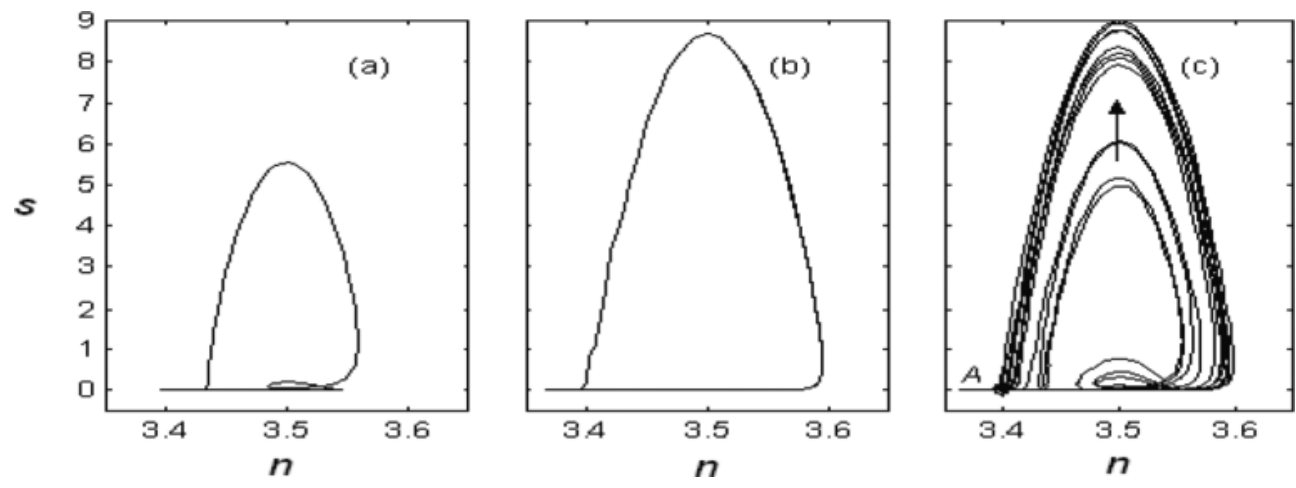

Figure 11. Influence of Gaussian noise on the evolution of the system in phases. 
Detailed numerical investigations have shown that for lower frequencies with respect to the relaxation frequency, system dynamics is periodic with the radiofrequency modulator signal period.

For a small enough modulation index $\left(m=\frac{I_{R F}}{I_{D C}}<<1\right)$, the periodic sinusoidal mode is obtained. The output power of the laser is sinusoidal, with the injection current (Figure 12a).

In the case of increasing of the modulation index and by approximating the frequency modulation with the frequency of the relaxation oscillations, a pulse operation mode (Figure 12b) is obtained, with a duration of the picoseconds (ultrashort pulses), the laser being used as a pulse source for optical communications.

For higher frequencies in relation to the resonance frequency of the system and modulation indices over a critical value, the phenomenon of doubling the frequency occurs.

The corresponding bifurcation diagrams are shown in [12]. At a value of parameter $m$ for which the dynamics is $2 \mathrm{~T}(n \mathrm{~T})$, the representation consists of two points ( $n$ points). Higher modulation frequencies cause multiple bifurcation points, including chaotic dynamics for certain parameter values $(f=8 \mathrm{GHz}$, in Figure 13).

Also in Figure 13, the dependence of the critical modulation index (frequency bifurcation) is observed (for $f=12 \mathrm{GHz}, \mathrm{m}_{\text {critic }}$ is higher than for $f=7 \mathrm{GHz}$ ). Figure 14 shows the laser nonlinear dynamics $4 \mathrm{~T}$ (Figure 14a) and the corresponding two-dimensional representation in the phase space (Figure 14b), for $I_{D C}=20 \mathrm{~mA}, f=8 \mathrm{GHz}$, and $m=3.5$. For an increased modulation index, $m=5.5$, the dynamics becomes chaotic (Figure $14 \mathrm{c}$ and $\mathbf{d}$ ).

The behavior is similar for other over-threshold injection current values, but there is an increasing dependence of the modulation index on doubling the period with the polarization current.

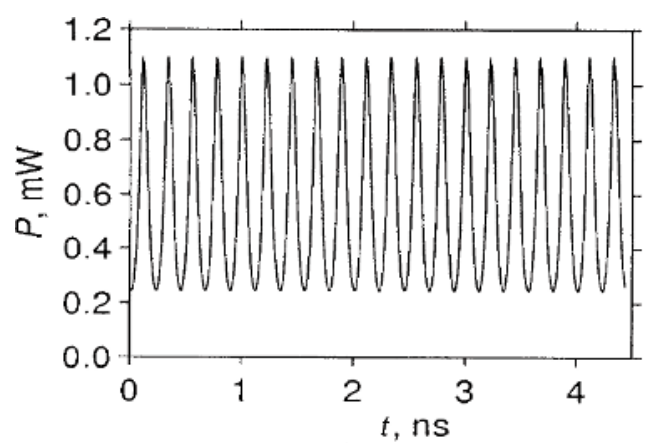

(a)

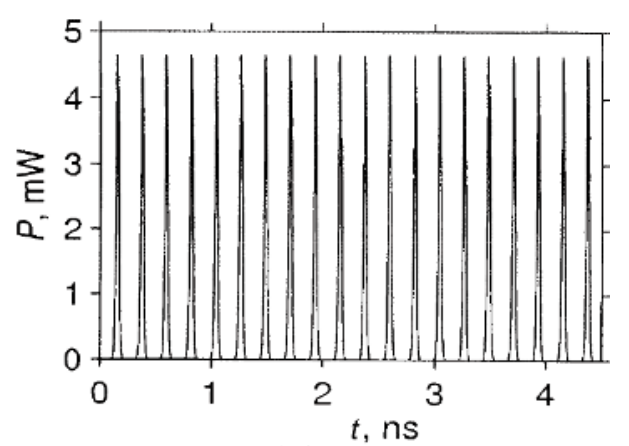

(b)

Figure 12. (a) Output power of the laser, sinusoidal for low signal modulation $(m=0,1)$; (b) the same pulse dependence, for $m=2(I=20 \mathrm{~mA}, f=4.5 \mathrm{GHz})$. 

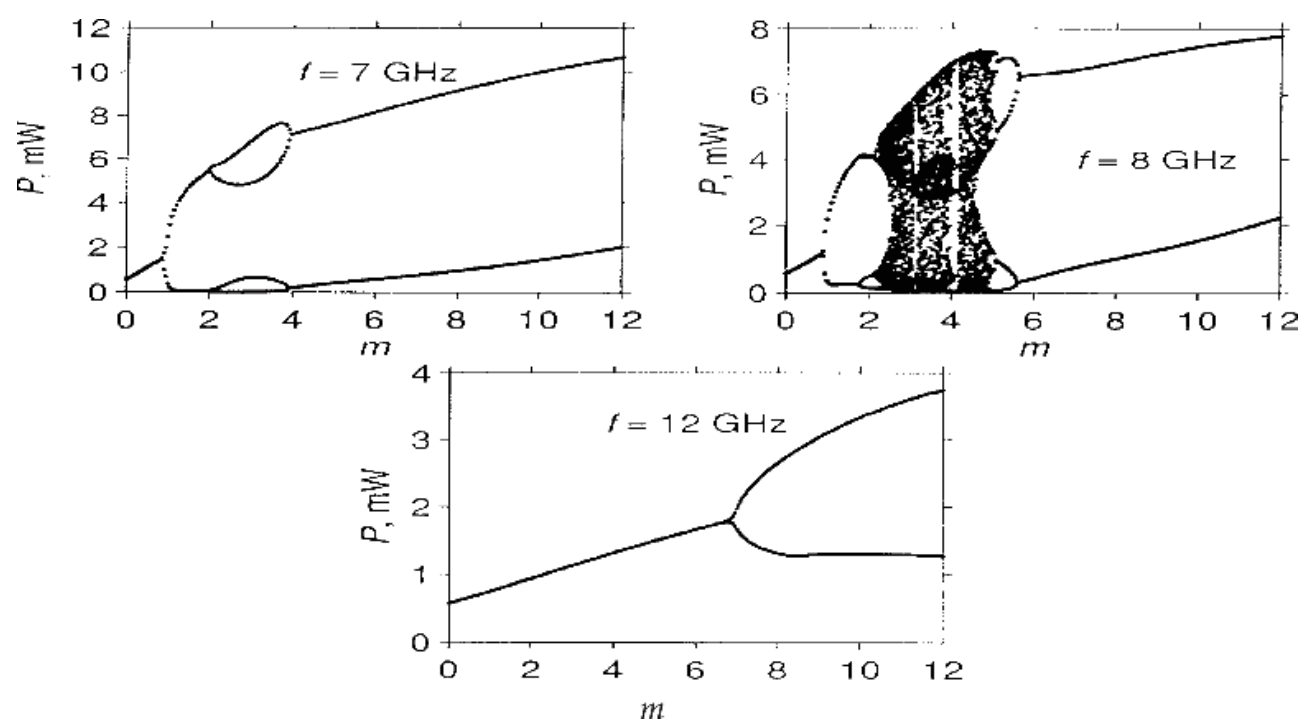

Figure 13. Bifurcation diagrams of the laser for different modulation frequencies.
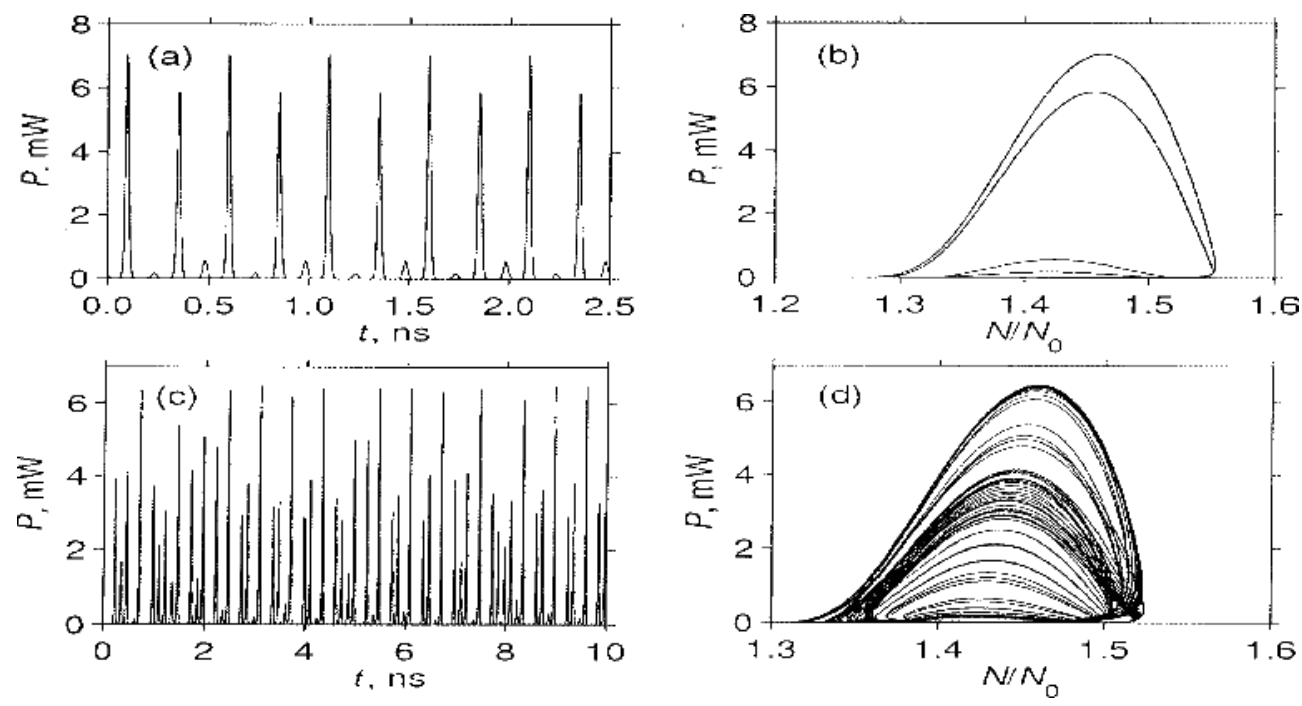

Figure 14. Nonlinear dynamics, temporal and in the phase space: $(a, b) 4 \mathrm{~T}$ for $\mathrm{m}=3.5$ and $(\mathrm{c}, \mathrm{d})$ chaotic for $\mathrm{m}=5.5$.

\section{Particularities of modeling and simulation of quantum well solar cells}

This part of the chapter is dedicated to research results concerning the simulation of optical parameters (refraction index, reflectance, and the absorption coefficient) of the quantum well 
(QW) photovoltaic cells, which represents the third generation of solar cells, proposed in 1990 by Keith Barnham [29,30], aiming to extend the spectral response and to increase the photocurrent of this kind of optoelectronics devices.

The quantum well solar cell is a p-i-n structure having quantum wells built in the intrinsic region. The doped parts on each side will produce an electric field perpendicular to the layers of quantum wells as is shown in [31]. The multiple quantum well solar cells (MQW) modeling is conducted at two levels dimensionally: a quantum level in which one computes the energy spectrum of the electron and the absorption coefficient of the MQW; a macroscopic level in which one studies the transport of charge carriers in a similar manner to the conventional p-i-n diode as in [30]. The reflection of light is brought, considering MQW cell a pseudohomogeneous medium. This combination of quantum and classical elements used in the modeling of MQW solar cell justifies the name of hybrid given to the model of MQW solar cell. An example of the application of the hybrid model $(\mathrm{HM})$ is given in [31]. It is the case of solar cell based on a ternary alloy semiconductor $\mathrm{A}_{\mathrm{x}} \mathrm{B}_{1-\mathrm{x}} \mathrm{C}\left(\mathrm{Al}_{\mathrm{x}} \mathrm{Ga}_{1-\mathrm{x}} \mathrm{As} / \mathrm{GaAs}\right)$ which has the lowest $\mathrm{BC}$ band gap $=0$ and achieves the greatest value $(\mathrm{x}=1)$ for the $\mathrm{AC}$ semiconductor band gap.

The hybrid model (HM) allows determining the configuration that leads to maximum conversion efficiency. This configuration can be designed, by using the transfer matrix method and the properties of the real materials. In other words, starting from a hypothetic system structure, the HM model allows optimization of the solar cell configuration in order to obtain maximum output electrical power, for a certain state of illumination as in [32].

\subsection{Modeling and simulation of the cell reflectance}

An important problem is to evaluate the effect of the quantum well number on the index of refraction and on the reflection losses, so the optimal number of the quantum well for the structure can be calculated [9].

In Figure 15, the results of the optical simulation of reflectance $R$ dependence on the radiation wavelength for different thicknesses $d$ of the anti-reflecting coating (ARC) are presented, in the case of anti-reflecting coating of $\mathrm{SiO}\left(n_{1}=1.4\right)$. Results obtained based on presented model are consistent with experimental data.

The simulation of refraction index and reflectance of the solar cells with quantum wells have been made with the Octave software, version 3.02. The cell reflectance can be calculated using the refraction indices of $\mathrm{GaAs}$ semiconductor and of the $\mathrm{Al}_{0.3} \mathrm{Ga}_{0.7} \mathrm{As}$ alloy as in [30]:

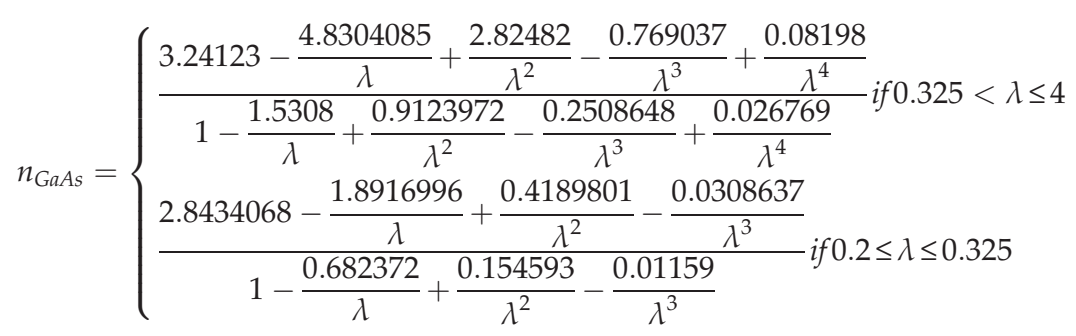




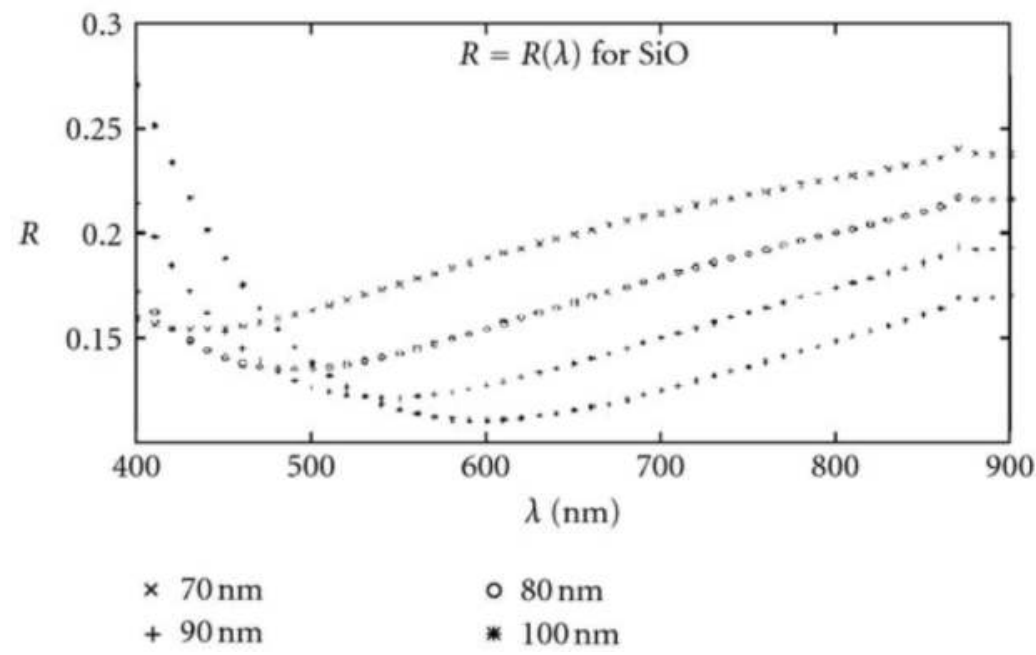

Figure 15. Simulation of the reflectance dependence of $\lambda$, for a solar cell QW structure with an $\mathrm{SiO}$ anti-reflecting coating. In Eq. (33), $\lambda$ is expressed in $\mu \mathrm{m}$. The refraction index of ternary alloy $\mathrm{Al}_{x} \mathrm{Ga}_{1-x} \mathrm{As}$ is calculated as.

$$
n_{A l_{x} G a_{1-x} A s}(\lambda)=\left[1.05-0.53 x+0.09 x^{2}\right] n_{G a A s}\left(\lambda_{x}\right)
$$

with $\lambda$ in $\mu \mathrm{m}$

To minimize the reflection losses, the solar cells are frequently designed with anti-reflecting coating (ARC).

The MQW layer was considered to consist of 30 quantum wells of GaAs of 20-nm width separated by barriers $\mathrm{Al}_{0.3} \mathrm{Ga}_{0.7} \mathrm{As}$ with a width of $10 \mathrm{~nm}$.

\subsection{Modeling and simulation of absorption coefficient}

In applications related to calculating the conversion efficiency of solar cells, but also in other situations, the coefficient of absorption (the absorbance) is practically described by continuous functions. For GaAs, according to the experimental data shown in [30], the following function that approximates the acceptable rate of absorption was determined:

$$
\begin{gathered}
\alpha_{\text {GaAs }}(\lambda)=\left\{\begin{array}{l}
e^{F_{1}(\lambda)}, 0.7<\lambda \leq 0.88 \\
e^{F_{2}(\lambda)}, 0.24<\lambda \leq 0.7 \\
e^{F_{2}(0.24)}, 0<\lambda \leq 0.24 \\
0, \text { otherwise }
\end{array}\right. \\
F_{1}(\lambda)=-0.7863+5.3115\left[1+\operatorname{erf}\left(-\frac{\lambda-0.84291}{0.038}\right)\right]
\end{gathered}
$$




$$
F_{2}(\lambda)=-447.432+4201.2 \lambda^{2}+6835.128 \lambda^{2} \ln \lambda-3781.193 \lambda^{3}+\frac{3.9049}{\lambda^{2}}
$$

In Ref. [30], the absorption coefficient for GaAs calculated with this model is represented.

\subsection{Simulation of the quantum efficiency of QW solar cells}

The analyzed model uses the transport equation of $\mathrm{Al}_{x} \mathrm{Ga}_{1-x} \mathrm{As}$ quantum well solar cells, where $x$ represents the aluminum concentration. The expression of the quantum efficiency $(Q E)$ is given by

$$
Q E \cong \frac{\alpha k(\alpha-S)}{\left(k^{2}-\alpha^{2}\right)(S \sinh (k z)-k \cosh (k z))} \cong \frac{\alpha-S}{\alpha^{2}(S-1)}
$$

where $\alpha$ is the spectral absorption, $\varepsilon_{f}$ is the effective electric field due to the minoritary carriers, and $S$ a parameter.

From the analysis of the data obtained, it results that quantum efficiency increases with any increase in $\lambda$, reaching significant values of a maximum of $90 \%$, in the case of large diffusion wavelength; quantum efficiency increases with any increase of $z$ and $\varepsilon_{f}$, respectively.

In [30] for the cell parameters $N_{w}=30, l_{w}=20 \mathrm{~nm}$ and $l_{b}=10 \mathrm{~nm}$, the calculation results of the conversion efficiency are summarized.

In Figure 16, it is observed that the conversion efficiency is strongly correlated with the number of quantum well up to $N w=30$; above this value, the efficiency is saturated at larger

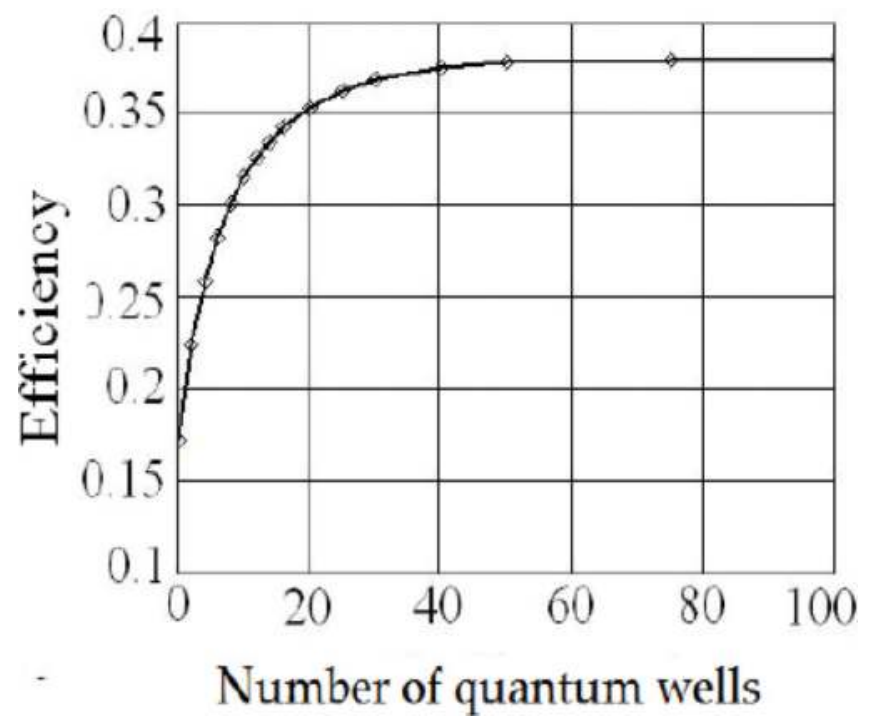

Figure 16. The dependence of the efficiency of MQW solar cell by the number of quantum wells. 
values of $N w$. The saturation is installed when the road length traveled by light is comparable with the absorption length. This result is a positive one by using the HM model. The calculation can be repeated varying different geometrical and material parameters in order to determine the optimal configuration, that is, one that maximizes efficiency.

Recently, good experimental results were reported in MQW solar cells. The fabricated solar cells based on $\operatorname{In}_{0.3} \mathrm{Ga}_{0.7} \mathrm{~N} / \mathrm{GaN}$ MQWs exhibit an open circuit voltage of about $2 \mathrm{~V}$, a fill factor of about $60 \%$, and an external efficiency of $40 \%$ at $420 \mathrm{~nm}$ and $10 \%$ at $50 \mathrm{~nm}$. New solar devices could be conceived based on optical properties of nanostructured materials $[29,30]$.

\section{Optical transmission system with laser chaotic carrier}

\subsection{The Pecora-Carroll synchronization method}

The method presented in this chapter focuses on the transmission of information by masking it in a chaotic signal, the amplitude of the message being added to that of the carrier. Two chaotic systems can be synchronized if they have similar parameters as is shown in Ref.s [35, 36]. This phenomenon has a very good potential application in coding the transmission of information. The transmission is made by using an emergent wave from a laser as a chaotic transmitter. The signal is attached to a carrier wave which is of a chaotic nature and has much higher amplitude. This ensures a higher degree of difficulty in intercepting and decoding. For attaching the signal to the carrier chaotic masking, modulation can be used. The properties of transmission and reception of the data as well as the synchronization of the lasers can be studied by using a pair of master-slave lasers. Modeling of the lasers is made by the use of the rate equations as in [35].

A communication scheme compatible with the Pecora-Carroll synchronization method given in $[36,40]$ is presented in Figure 17. The link between the master system and the slave subsystems in the transmission area is unidirectional. The encryption is done by using the chaotic signal of the slave system at the transmitter as carrier for the message. At the receiver, the slave system synchronizes with its replica at the transmitter through the one linking drive signal. This allows the extraction of the information from the total transmitted signal.

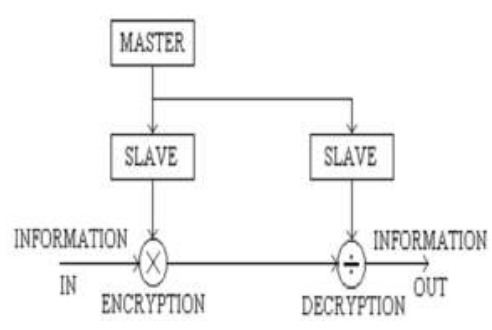

Figure 17. Block scheme of a communication system based on the synchronization of two chaotic subsystems. 


\subsection{Optical communications channel with chaotic laser carrier}

It was shown that an amplitude modulator-demodulator with chaotic carrier can be implemented using a pair of self-pulsating laser diode (SPLDs), with closely matched parameters. If a sinusoidal injection current is superimposed over a polarization current for which selfpulsations occur, then the pulses become chaotic both in amplitude and in repetition interval. When the sinusoidal current is modulated with an information signal having amplitude and frequency much smaller than the disorder maker current, then the transmitted signal spectrum does not present a clear component having the frequency of the message, and the filtering operation is of no use in recovering the transmitted information. If a small part of the transmitted chaotic signal (SPLD-T) is coupled into the received signal (SPLD-R), the information can be recovered based on the property that the receiver output field synchronizes only with the chaotic carrier and not with the whole transmitted optical field. An SPLD is an active optical device able to produce a continuous train of pulses with repetition rate dependent on the injection current (Figure 18).

The self-pulsating laser diode (SPLD) is driven by an injection current $I^{\mathrm{T}, \mathrm{R}}(t)$ :

$$
I^{\mathrm{R}, \mathrm{T}}=I_{\text {bias }}^{\mathrm{R}, \mathrm{T}}+I_{\mathrm{A}}^{\mathrm{R}, \mathrm{T}} \sin (2 \pi f t)
$$

where $I_{\text {bias }}$ is the continuous component of the injection current, while $I_{\mathrm{A}}$ and $f$ are the amplitude and frequency of a sinusoidal current superimposed over the continuous injection bias component, respectively. The laser parameters for numerical simulations can be found in [10]. It is noted that $\delta$ is the coupling factor of the transmitted field into the receiver SPLD.

If a sinusoidal current is superimposed over $I_{\text {bias }}$, then the self-pulsations became chaotic either in repetition interval or in amplitude (Figure 19). The two identical SPLD-R and SPLD-T have similar outputs but uncorrelated [35].

When a small part of the transmitted chaotic carrier is coupled into the receiver device, synchronization between the two lasers becomes possible (Figures 20 and 21), independently of the initial conditions of the two systems.

To investigate the possibility of transmission information, we introduce the information as an amplitude modulation of the sinusoidal injection current into the transmitter laser. For simplicity,

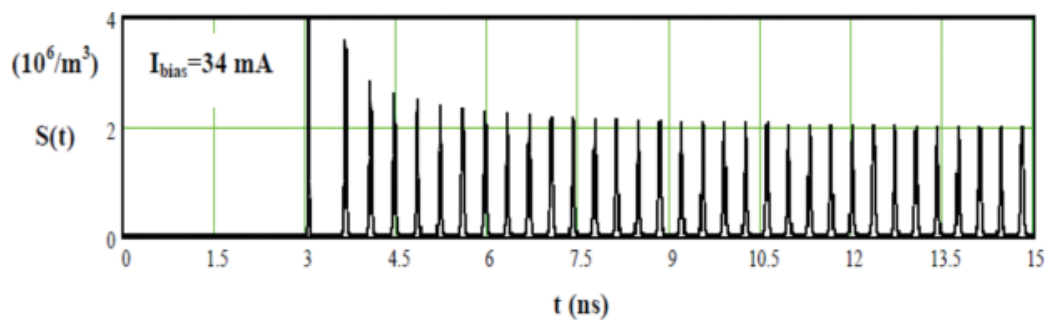

Figure 18. Temporal dynamics of SPLD for the injection current $I_{\text {bias }}=34 \mathrm{~mA}$. 


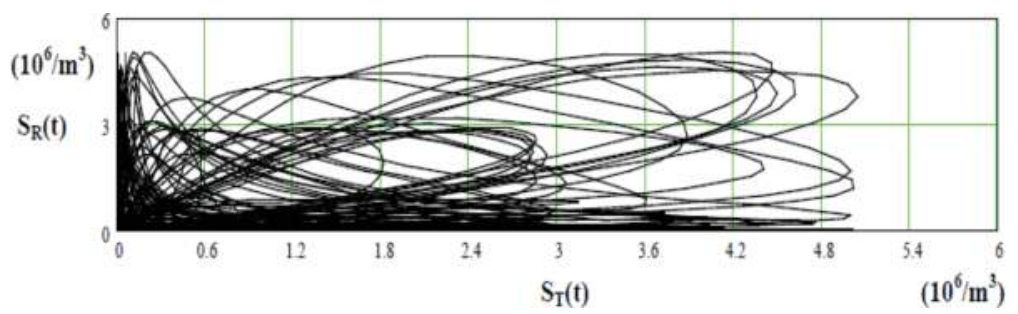

Figure 19. The output $S_{\mathrm{R}}(t)$ of SPLD-R versus $S_{\mathrm{T}}(t)$ SPLD-T. The two photon densities are not synchronized.

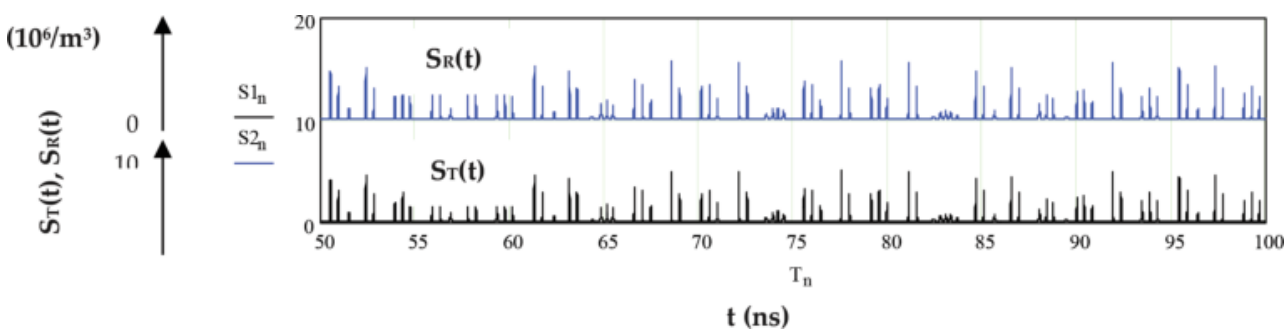

Figure 20. The graphical representation of the photon densities $S_{\mathrm{R}}(t)$ and $S_{\mathrm{T}}(t)$, synchronized, for $\delta=0.04$.

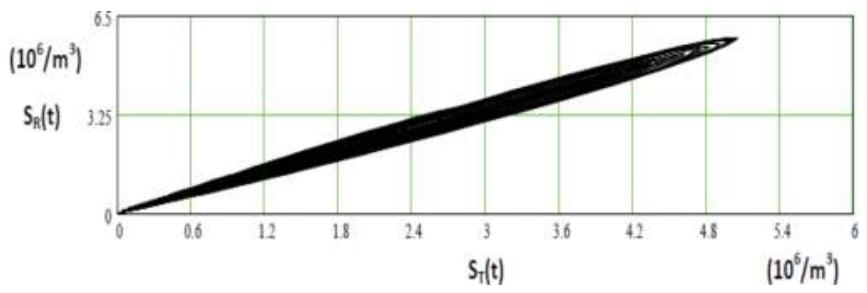

Figure 21. The output $S_{\mathrm{R}}(t)$ of SPLD-R versus $S_{\mathrm{T}}(t)$ SPLD-T. The two photon densities are closely synchronized.

we consider a sinusoidal message signal $I_{\mathrm{m}}(t)=I_{\mathrm{m}, \mathrm{A}} \sin \left(2 \pi f_{\mathrm{m}} t\right)$, where $I_{\mathrm{m}, \mathrm{A}}<<I_{\text {bias }}$ and $f_{\mathrm{m}}<<f$ for secrecy and small modulation distortion reasons. Numerical simulations show that the receiver output synchronizes with the carrier field, rather than to the transmitted signal; the decoded signal is obtained as $S_{d}(t)=S_{T}(t)-S_{R}(t)$ In Figure 22, a typical method of recovering information in such a kind of transmission is presented.

The useful message can be recovered by low filtering of the quantity $\left[S_{T}(t)-S_{R}(t)\right] / S_{R}(t)$. As SPLDs are widely available, cheap, and compact, their use in private communication systems, but not only, for transmissions with information encrypted is recommended. 


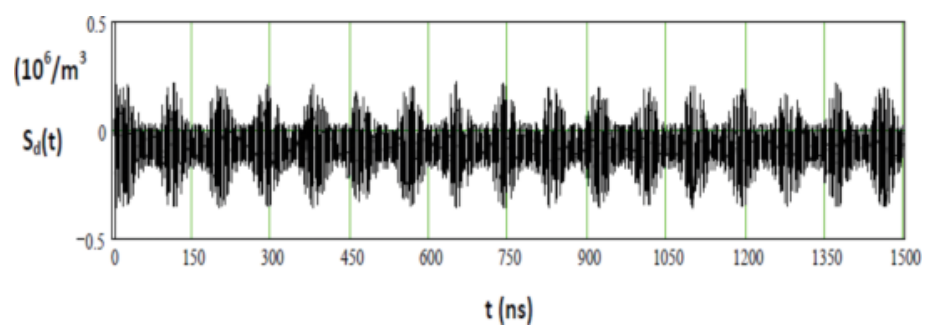

Figure 22. The decoded signal versus time. $S_{d}(t)=\frac{S_{T}(t)-S_{R}(t)}{S_{R}(t)}$ for $I_{\mathrm{m}}(t)=(7 / 50) \sin [2 \pi(f / 50) t] \mathrm{mA}$.

\section{Conclusions}

The mathematical models presented in this chapter highlight the possibility of unitary, describing the nonlinear dynamics in the optoelectronics structures with quantum well fact which is essential for their design and applications.

The obtained results by numerical simulations show how the dynamics is characterized by bifurcation points and chaos, being close to those obtained experimentally and reported in the literature.

The results based on the hybrid model of the MQW solar cells show that the number of quantum well of the structure can be determined by numerical simulations for improving the conversion efficiency, based on optical properties of nanostructured materials used in research and fabrication.

The broadband modulation and the low injection current at the oscillation threshold of the quantum well lasers compared to bulk laser ones recommends these optoelectronics structures for high-speed optical communications, providing unwanted nonlinear effects as the points of bifurcation and chaos are avoided, for the stable operation and the control of their dynamics.

\section{Conflict of interest}

The authors declare that there is no conflict of interest regarding the publication of this chapter.

\section{Author details}

\section{Andreea Rodica Sterian}

Address all correspondence to: andreea.sterian1@yahoo.com

Faculty of Applied Sciences, Physics Department, Academic Center of Optical Engineering and Photonics, Polytechnic University of Bucharest, Bucharest, Romania 


\section{References}

[1] Weiss U. Quantum Dissipative Systems. Toh Tuck Kink, Singapore: World Scientific Publishing Co. Pte.Ltd.; 2012

[2] Sterian PE. Analytical model of dissipative coupling coefficients in laser structures. Proceedings of the Romanian Academy A. 2002;3(3):123-126

[3] Peter SZ, Zory J. Quantum Well Lasers. Vol. 347. Boston: Academic Press; 1993

[4] Sterian P. Exact quantum master equations for Markoffian systems. Optical Engineering. 1996 Jun 1;35(6):1573-1575. DOI: 10.1117/1.601001

[5] Sterian PE. Fotonica. Printech; 2000

[6] Stefanescu E, Sterian AR, Sterian P. Study on the fermion systems coupled by electric dipol interaction with the free electromagnetic field. In: 21st European Mask and Lithography Conference 2005 Jun 7 (pp. 160-165). International Society for Optics and Photonics. DOI: $10.1117 / 12.63368$

[7] Sterian AR. Coherent radiation generation and amplification in erbium doped systems. In: Advances in Optical Amplifiers. Croatia: InTech; 2011

[8] Iordache DA, Sterian P, Sterian AR, Pop F. Complex computer simulations, numerical artifacts, and numerical phenomena. International Journal of Computers Communications \& Control. 2010 Dec 1;5(5):744-754. DOI: 10.15837/ijccc.2010.5.2234

[9] Barnham KW, Duggan G. A new approach to high-efficiency multi-band-gap solar cells. Journal of Applied Physics. 1990 Apr 1;67(7):3490-3493. DOI: 10.1063/1.345339

[10] Sterian AR. Amplificatoare optice. Editura Printech; 2006. DOI: 10.1109/3.748833

[11] Ghoniemy S, MacEachern L, Mahmoud S. Extended robust semiconductor laser modeling for analog optical link simulations. IEEE Journal of Selected Topics in Quantum Electronics. 2003 May;9(3):872-878. DOI: 10.1109/JSTQE.2003.818841

[12] Sterian AR. Numerical simulations on nonlinear dynamics in lasers as related high energy physics phenomena. Advances in High Energy Physics. 2013 Oct 27;2013. DOI: $10.1155 / 2013 / 516396$

[13] Sterian AR. The structure of an automatic decision system for a large number of independent particle detectors. Advances in High Energy Physics. 2013 Mar 10;2013. DOI: 10.11 $55 / 2013 / 839570$

[14] Anghel DA, Sterian AR, Sterian PE. Modeling quantum well lasers. Mathematical Problems in Engineering. 2012 Mar 7;2012. DOI: 10.1155/2012/736529

[15] Tsou BP, Pulfrey DL. A versatile SPICE model for quantum-well lasers. IEEE Journal of Quantum Electronics. 1997 Feb;33(2):246-254. DOI: 10.1109/3.552265

[16] Dehghan M, Derakhshan P. A novel dynamic analysis and simulation for quantum-well distributed feedback laser,(qw-dfb). Progress in Electromagnetics Research B. 2008;3:105-114 
[17] Popescu IM, Preda AM, Tudorache SS. Aplicatii ale laserilor. Bucuresti: Tehnica; 1979

[18] Gao DS, Kang SM, Bryan RP, Coleman JJ. Modeling of quantum-well lasers for computeraided analysis of optoelectronic integrated circuits. IEEE Journal of Quantum Electronics. 1990 Jul;26(7):1206-1216. DOI: 10.1109/3.59660

[19] Javro SA, Kang SM. Transforming Tucker's linearization laser rate equations to a form that has a single solution regime. Journal of Lightwave Technology. 1995 Sep;13(9):1899-1904. DOI: $10.1109 / 50.464741$

[20] Mena PV, Kang SM, DeTemple TA. Rate-equation-based laser models with a single solution regime. Journal of lightwave Technology. 1997 Apr;15(4):717-730. DOI: 10.1109/ 50.566695

[21] Sterian AR. Computer modeling of the coherent optical amplifier and laser systems. In: International Conference on Computational Science and Its Applications 2007 Aug 26. Springer, Berlin, Heidelberg. pp. 436-449

[22] Bulinski M, Pascu ML. Chaos in laser diode light emission. Romanian Journal of Optoelectronics. 2001 Apr;9(2):1-34

[23] Lee $\mathrm{CH}$, Yoon TH, Shin SY. Period doubling and chaos in a directly modulated laser diode. Applied Physics Letters. 1985 Jan 1;46(1):95-97. DOI: 10.1063/1.95810

[24] Bennett S, Snowden CM, Iezekiel S. Nonlinear dynamics in directly modulated multiplequantum-well laser diodes. IEEE Journal of Quantum Electronics. 1997 Nov;33(11):2076-2083. DOI: $10.1109 / 3.641323$

[25] Ninulescu V, Nicolae VB, Sterian A. Quantum well lasers for medical industry. InBIRD. 2008. pp. 563-570

[26] Nagarajan R, Ishikawa M, Fukushima T, Geels RS, Bowers JE. High speed quantum-well lasers and carrier transport effects. IEEE Journal of Quantum Electronics. 1992 Oct;28(10): 1990-2008. DOI: 10.1109/3.159508

[27] Marcuse DI. Computer simulation of laser photon fluctuations: Theory of single-cavity laser. IEEE Journal of Quantum Electronics. 1984 Oct;20(10):1139-1148. DOI: 10.1109/ JQE.1984.1072276

[28] Sterian AR, Ninulescu V, Sterian L. Modulated laser diode for medical applications. In: Proc. of SPIE Vol 2004 Oct 21 Vol. 5581. p. 275. DOI: 10.1117/12.582837

[29] Barnham KW, Barnes JM, Braun B, Connolly JP, Haarpaintner G, Nelson JA, Paxman M, Button C, Roberts JS, Foxon CT. A novel approach to higher efficiency-the quantum well solar cell. In: Proceedings of the 11th EC Photovoltaic Solar Energy Conference 1992 pp. 146-149

[30] Fara L, editor. Advanced Solar Cell Materials, Technology, Modeling, and Simulation. IGI Global; 2012 Jul 31

[31] Fara S, Sterian P, Fara L, Iancu M, Sterian A. New results in optical modelling of quantum well solar cells. International Journal of Photoenergy. 2012 May 16:2012. DOI: 10.1155/ 2012/810801 
[32] Fara L, Moraru AG, Sterian P, Bobei AP, Diaconu A, Fara S. Building integrated photovoltaic (BIPV) systems in Romania. Monitoring, modelling and experimental validation. Journal of Optoelectronics and Advanced Materials. 2013 Jan 1;15(1-2):125-130

[33] Iordache DA, Sterian PE, Tunaru I. Charge coupled devices as particle detectors. Advances in High Energy Physics. 2013 Mar;19:2013. DOI: 10.1155/2012/810801

[34] Popescu DG, Sterian P. Photonic crystal fiber mode characterization with multipole method. UPB Scientific Bulletin, A Series. Bucharest: University "Politehnica" of Bucharest; 2013 Jan 1;75:205-215

[35] Sterian P, Ninulescu V, Sterian AR, Lazar B. Optical communication methods based on chaotic laser signals. University Politehnica of Bucharest scientific bulletin-series Aapplied mathematics and. Physics. 2010 Jan 1;72(1):83-94

[36] Pecora LM, Carroll TL. Driving systems with chaotic signals. Physical Review A. 1991 Aug 1;44(4):2374. DOI: 10.1103/PhysRevA.44.2374

[37] Sterian PE. Transmisia optica a informatiei. Bucharest: Editura Tehnică; 1981

[38] FAZACAŞ A, Sterian P. Propagation of the Raman soliton in optical fibers. Romanian Reports in Physics. 2013;65(4):1420-1430

[39] Sanchez-Diaz A, Mirasso CR, Colet P, Garcia-Fernandez P. Encoded Gbit/s digital communications with synchronized chaotic semiconductor lasers. IEEE journal of quantum electronics. 1999 Mar;35(3):292-297

[40] Sterian PE. Communication based on chaotic signals. Proceedings of the Romanian Academy. 2002;3(1-2):45-48 